(C) 2016 IEEE. Personal use of this material is permitted. Permission from IEEE must be obtained for all other uses, in any current or future media, including reprinting/republishing this material for advertising or promotional purposes, creating new collective works, for resale or redistribution to servers or lists, or reuse of any copyrighted component of this work in other works. 


\title{
On the Achievable Throughput of Cooperative Vehicular Networks
}

\author{
Jieqiong Chen*, Ammar Zafar*, Guoqiang Mao*†‡§, Changle Li \\ ${ }^{*}$ School of Computing and Communication, The University of Technology Sydney, Australia \\ ${ }^{\dagger}$ Data61 Australia \\ ${ }^{\ddagger}$ Beijing University of Posts and Telecommunications, Beijing, China \\ ${ }^{\S}$ School of Electronic Information Communications, Huazhong University of Science Technology, Wuhan, China \\ IState Key Laboratory of Integrated Services Networks, Xidian University, Xi'an, Shaanxi, 710071 China \\ Email: jieqiong.chen@student.uts.edu.au, ammar.zafar@uts.edu.au,g.mao@ieee.org, clli@mail.xidian.edu.cn
}

\begin{abstract}
Due to the time-varying channel conditions and dynamic topology of vehicular networks attributable to the high mobility of vehicles, data dissemination in vehicular networks, especially for content of large-size, is challenging. In this paper, we propose a cooperative communication strategy for vehicular networks suitable for dissemination of large-size content and investigate its achievable throughput. The proposed strategy exploits the cooperation of vehicle-to-infrastructure (V2I) communications, vehicle-to-vehicle (V2V) communications and the mobility of vehicles to facilitate the transmission. Detailed analysis is provided to characterize the data dissemination process using this strategy and a closed-form result is obtained on its achievable throughput, which reveals the relationship between major performance-impacting parameters such as distance between infrastructure, radio ranges of infrastructure and vehicles, transmission rates of V2I and V2V communications and vehicular density. Simulation and numerical results show that the proposed strategy significantly increases the throughput of vehicular networks even when the traffic density is low. The result also gives insight into the optimum deployment of vehicular network infrastructure to maximize throughput.

Index Terms-Data dissemination, cooperative communication, throughput, vehicular networks, vehicle-to-infrastructure, vehicle-to-vehicle.
\end{abstract}

\section{INTRODUCTION}

Vehicular networks have recently gained significant attention from academia and industry because of their increasingly important role in enhancing road traffic safety and providing useful information to drivers and passengers [1]- [3]. By disseminating real-time information about accidents, traffic congestion or obstacles in the road, traffic safety and efficiency can be largely improved. Furthermore, offering information like digital maps with real-time traffic status and in-car entertainment services greatly enhance the convenience and comfort of drivers and passengers.

There are two major data dissemination techniques in vehicular networks, i.e., vehicle-to-infrastructure (V2I) and vehicleto-vehicle (V2V) communications. V2V communications such as VANET, on one hand is easy to deploy, suitable for real-time communication between nearby vehicles and offers cost-effective solutions to vehicular communications. On the other hand, it is well known that $\mathrm{V} 2 \mathrm{~V}$ communications may become unreliable [4] and incur long communication delay for communications when the vehicular density is low [5]. Thus, infrastructure support in the form of Road-Side Units (RSU) or LTE base stations is essential and beneficial. However, due to the relatively high cost of deployment, infrastructure communications, e.g., V2I communications can be limited, especially in rural areas and in the initial deployment phase of vehicular networks. Besides, each individual vehicle's sojourn time within one infrastructure point's coverage may be short due to the high speed of vehicles and limited coverage of infrastructure. This consequently may lead to vehicles' failure to download a large-size file during their sojourn when relying purely on V2I communications. Therefore V2I and V2V communications may have to co-exist and complement with each other to meet the diverse communication requirements in vehicular networks ranging from safety information dissemination to in-car entertainment services.

Cooperative communication has been widely applied in vehicular networks and has numerous advantages (e.g., increase downloaded data volume [6], reduce transmission delay [7], improve spectral efficiency [8] etc.), as well as some unique and significant challenges. First, caused by the high mobility of vehicles, the topology of vehicular network changes frequently, which consequently leads to frequent fragmentation and disconnectivity of $\mathrm{V} 2 \mathrm{I}$ and $\mathrm{V} 2 \mathrm{~V}$ communications. In addition, vehicular wireless channels may become lossy due to fading and path loss which in turn affects the reliability of V2I and V2V communications. Furthermore, it is well known that "mobility improves throughput" [9] and this is particularly true in vehicular networks. Therefore, how to optimally design cooperative communication scheme that utilizes the mobility of vehicles to carry information and maximizes throughput for those services that can tolerate large delay is both an interesting and challenging topic.

In this paper, we propose a novel cooperative communication strategy that utilizes V2I communications, V2V communications and the mobility of vehicles to improve the throughput of vehicular networks. Specifically, using the proposed cooperative communication strategy, when a vehicle of interest (VoI) moves into the coverage of infrastructure, it receives data from the infrastructure via V2I communications. When the VoI moves outside the coverage of infrastructure, it utilizes 
V2V communications with vehicles traveling in the opposite direction (defined as helpers), their mobility, and helpers' V2I communications to continue receiving data to boost its throughput. In this way, V2I communications between a VoI and infrastructure, between helpers and infrastructure, V2V communications and vehicular mobility are coherently combined to maximize the throughput of a VoI. We analyze the throughput achievable by a VoI using this scheme. Through the analysis, the relationship between the major performanceimpacting parameters is characterized, which shed insight into the optimum deployment of vehicular network infrastructure.

The main contributions of this paper are given as follows:

1) a cooperative communication strategy is proposed, which exploits the cooperation of V2I communications, $\mathrm{V} 2 \mathrm{~V}$ communications and the mobility of vehicles to improve the achievable throughput.

2) an analytical framework is proposed for studying the data dissemination process under our cooperative communication strategy and a closed-form expression of the achievable throughput is obtained.

3) simulation and numerical results validate the significant benefit of the cooperative communication scheme and shows that even when traffic density is rather low, the cooperative strategy can still greatly enhance the performance of the system in terms of throughput.

The rest of this paper is organised as follows: Section II reviews related work. Section III introduces the network and system model, the proposed cooperative communication scheme and the problem formation. The theoretical analysis is given in Section IV. In Section V, we validate the analytical results using simulations and discuss the performance. Section VI concludes this paper.

\section{RELATED WORK}

There have been extensive studies on improving the performance (e.g., throughput, downloaded data volume, communication link quality etc.) of vehicular networks. Among all the key techniques to enhance these performance measures, cooperative communication is a popular and important one, which usually includes cooperation among vehicles and separate cooperation within infrastructure. Our work combines both kinds of cooperation and exploits the performance benefits of vehicular mobility too.

In terms of vehicular cooperation, Zhou et al. [6] introduced a cooperative approach by forming a linear vehicle cluster on the highway to cooperatively download the same content file from the infrastructure to enhance the probability of successful file download. In [7], Zhu et al. studied multiple vehicles approaching each other collaboratively download data from an RSU by using network coding and analysed the average download time. In both aforementioned works [6], [7], vehicular cooperation only occurs when vehicles have a common interest to download the same file from the infrastructure, which is different from our work that focuses on multiple vehicles cooperating to deliver a file to a VoI utilizing V2I, $\mathrm{V} 2 \mathrm{~V}$ communications and the mobility of vehicles.
Infrastructure cooperation is achieved by caching different files in different infrastructure points to help moving vehicles get more valid content. In [1] and [10], by utilizing infrastructure cooperation for data dissemination, the authors investigated the optimal cooperative content dissemination scheme in vehicular networks to maximize disseminated data size [1] and data dissemination success probability [10] respectively. However, both [1] and [10] only considered V2I communications.

Compared with the above work, this paper focuses on the achievable throughput by a VoI when V2I communications, V2V communications and vehicular mobility can all be exploited to improve the throughput. This forms an interesting and important scenario in vehicular networks which, to the best of our knowledge, has not been studied before.

\section{SYSTEM MODEL}

\section{A. Network Model}

We consider a highway scenario with bi-directional flows where the highway is modeled by an infinite line with roadside infrastructure, e.g., RSUs or LTE base stations, regularly deployed with equal distance of $d$. The width of a lane is typically small compared with the transmission range. Therefore multiple lanes in the same direction can be abstracted as a single lane [11]- [13]. We further assume that infrastructure points are connected to the backbone network through wired or wireless links so that they can provide information services to vehicles within their coverage.

We adopt a commonly used traffic model that vehicles in each direction are Poissonly distributed with mean density $\rho_{1}$ $\mathrm{veh} / \mathrm{m}$ and $\rho_{2} \mathrm{veh} / \mathrm{m}$ respectively, and consequently the intervehicle distance follows an exponential distribution [5], [12], [14]. Similar to previous works [5], [12], [15], we assume that vehicles in each direction move at a constant speed of $v_{1}$ and $v_{2}$ respectively. Individual vehicular speed may deviate slightly from the mean speed. However, such deviations are expected to have very minor impact on the throughput being studied. The system model is illustrated in Fig. 1.

\section{B. Wireless Communication Model}

As introduced earlier, we consider two types of wireless communication schemes: V2I and V2V communications. We assume that each vehicle has a single antenna so that they can not transmit and receive at the same time. Each infrastructure and each vehicle has the same transmission range, denoted by $r_{I}$ and $r_{0}$ respectively and $r_{I}>r_{0}$, which reflects the fact that infrastructure typically has stronger communication capability. Vehicle and vehicle (infrastructure) can directly communicate with each other if and only if their Euclidean distance is no larger than the transmission range $r_{0}\left(r_{I}\right)$. This simplified unit disk model has been extensively used in the field [5], [13], [16]. It grossly captures the fact that all wireless devices have a limited transmission range and that the closer two devices become, the easier it is for them to establish a connection. This simplification allows us to omit physical layer details and focus on the topological impact of vehicular networks on 


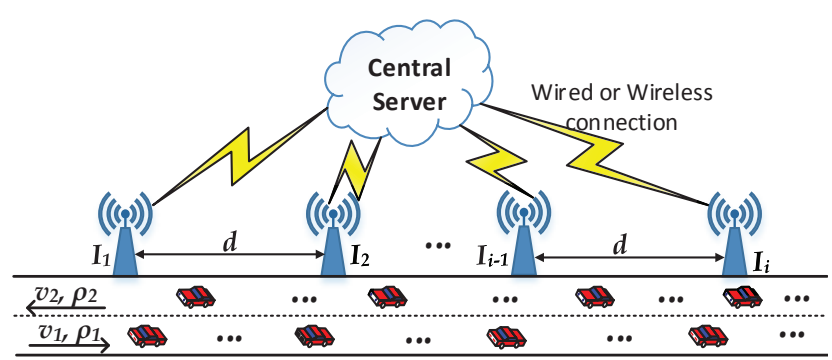

Fig. 1. An illustration of the system model for a bi-directional highway with infrastructure regularly deployed with equal distance of $d$. The density and speed of vehicles in each lane are $\rho_{1}, v_{1}$ and $\rho_{2}, v_{2}$ respectively.

throughput, and we will show later in the simulation that our analysis assuming the unit disk model can be extended to a more realistic log-normal shadowing model.

We assume that V2I and V2V communicate at a constant data rate $w_{I}$ and $w_{V}$ respectively and $w_{I}>w_{V}$. It is because that V2I communications usually are allocated to larger bandwidth and signal power compared to $\mathrm{V} 2 \mathrm{~V}$ communications so that they can provide larger transmission rate. This constant rate assumption also helps to omit the impact of physical layer details. For time-varying channels, the values of $w_{I}$ and $w_{V}$ can be replaced by the respective time-averaged throughput of V2I and V2V communications and our analysis still applies. This has been validated later using simulations.

Furthermore, we assume that each infrastructure has $N$ channels so that at most $N$ vehicles can simultaneously receive data from the same infrastructure. Once a vehicle gains access to a channel, it will occupy it until it leaves the coverage of the infrastructure. It is assumed that V2I communications have higher priority than $\mathrm{V} 2 \mathrm{~V}$ communications, which means when a vehicle is receiving data from infrastructure, it can not transmit (receive) data to (from) other vehicles. This assumption can be justified by the fact that V2I communication provides a higher data rate than $\mathrm{V} 2 \mathrm{~V}$ communication.

\section{Cooperative Communication Strategy}

Specially, in this paper we consider a scenario where a VoI wants to download a large file, e.g., a video, from a remote server. Without loss of generality, we assume that the VoI travels at speed $v_{1}$ and the vehicles in the opposite direction (recall that we define them as helpers) travel at speed $v_{2}$ and have vehicular density $\rho_{2}$.

A cooperative communication scheme is designed to utilize the V2I communications, V2V communications and mobility of vehicles to facilitate the transmission. The large file the VoI interests may be first split into multiple pieces and transmitted to different infrastructure points so that they have different pieces of data, which achieves cooperation among infrastructure. Data delivered to infrastructure may be further split and transmit to the $\mathrm{VoI}$ and different helpers when they move into the coverage so that each helper has different pieces of data from each other and from the VoI, and these data will be transmit to the VoI when they are encounter, which exploits the mobility of vehicles and $\mathrm{V} 2 \mathrm{~V}$ communications to achieves the vehicular cooperation.

Particularly we consider two consecutive infrastructure points along the travel direction of the VoI collaborate to deliver the file. Denote the nearest infrastructure point by $I_{1}$ and the second nearest one by $I_{2}$. When the $\mathrm{VoI}$ is in the coverage of $I_{1}$, it receives data directly from $I_{1}$. When the VoI moves outside the coverage of $I_{1}$, it may continue to receive data from helpers using $\mathrm{V} 2 \mathrm{~V}$ communications as each helper has received different pieces of data from $I_{2}$. Here only one-hop V2V communications between the VoI and helpers is considered. Of course, when the VoI moves along its direction, the two infrastructure points participating in the cooperative communication are also updated. In this way, V2I communications between the VoI and infrastructure, between helpers and their respective infrastructure points, $\mathrm{V} 2 \mathrm{~V}$ communications between the $\mathrm{VoI}$ and helpers as well as vehicular mobility are coherently combined to maximize the throughput of the VoI. Furthermore, we consider some practical issues like out of sequence data delivery can be handled by techniques such as network coding (e.g., our recent paper [17]) so that we can focus on the main theme of the paper without the need for considering their impact.

Consider an arbitrarily chosen time interval $[0, t]$, denote the amount of data received by the $\mathrm{VoI}$ as $D(t)$, which includes both data received from infrastructure and helpers. In this paper, we are interested in finding the long-term throughput of the VoI using our cooperative communication strategy where the throughput is formally defined as follows:

$$
\eta=\lim _{t \rightarrow \infty} \frac{D(t)}{t} .
$$

\section{TheOretiCAL ANALYSis}

In this section, we will present detailed theoretical analysis of the data dissemination process in our system model.

\section{A. Problem Transformation}

We define the period from the VoI enters the coverage of one infrastructure to the VoI enters the coverage of the next infrastructure as one cycle. It follows from the above definition that the total amount of data the VoI receives in each cycle is correlated rather than independent. This is due to our assumption that each infrastructure has $N$ channels. Thus, the amount of data each helper obtains from the infrastructure is dependent on its previous (along its travel direction) $N$ vehicles' inter-vehicle distance (we will show this dependence later in Section IV.C as equation (4)). As the data the VoI receives during $\mathrm{V} 2 \mathrm{~V}$ communications comes from the data received by the helpers during their V2I communications, the correlation between helpers' received data amount causes the correlation between data amount the VoI receives from each cycle.

If we approximately omit this correlation (we will show later in the simulation that this approximation causes negligible impact on our results), then the entire data receiving 


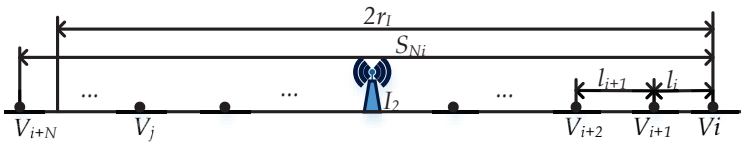

(a). $n \leq N-1$, vehicle $V_{i+N}$ is outside $I_{2}$ 's coverage

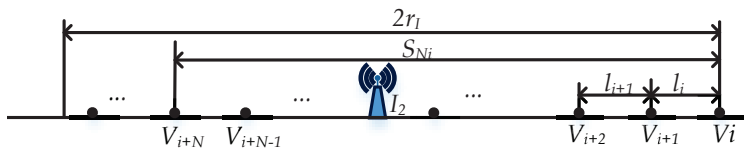

(b). $n>N-1$, vehicle $V_{i+N}$ is within $I_{2}$ 's coverage

Fig. 2. An illustration of helpers' V2I communication model.

process of the VoI can be regarded as a renewal reward process [18], where each cycle consists one V2I communication process, followed by a $\mathrm{V} 2 \mathrm{~V}$ communication process, and the reward is the data amount the VoI receives during each cycle. Thus, the achievable throughput in our system can be equally calculated as follows:

$$
\eta=\lim _{t \rightarrow \infty} \frac{D(t)}{t}=\lim _{t \rightarrow \infty} \frac{\int_{0}^{t} D(\tau) d \tau}{t}=\frac{E\left[D_{I}\right]+E\left[D_{V}\right]}{d / v_{1}}
$$

where $E\left[D_{I}\right]$ and $E\left[D_{V}\right]$ are the expected amount of data the VoI receives from infrastructure and helpers in one cycle respectively.

\section{B. V2I Communications}

As explained in Section III.C, when the VoI is covered by an infrastructure point, it will only use V2I communications. Therefore, the expected amount of data the VoI can receive from infrastructure in one cycle is

$$
E\left[D_{I}\right]=\frac{2 r_{I} w_{I}}{v_{1}}
$$

\section{V2V Communications}

Without loss of generality, we denote during one cycle, the infrastructure participating in the VoI's V2I communication process by $I_{1}$ and the other infrastructure participating in helpers' V2I communication process by $I_{2}$. Besides, we designate the time instant when the VoI leaves the coverage of $I_{1}$ as $t=0$ and define its moving direction as the positive (right) direction of the coordinate system and at $t=0$, point in the left of the $\mathrm{VoI}$ and with a distance of $r_{0}$ to the $\mathrm{VoI}$ is set to be the origin.

In the following, we first show that our problem can be converted into a coverage process problem and then analytically solve the converted problem.

1) Coverage Process Problem Conversion: As a helper must first receive data from an infrastructure point before delivering the data to the VoI, it may likely occur that a helper runs out of data (the helper is still within the VoI's coverage, but it has no data to transmit to the VoI) during their encounter. The following proposition shows that such scenario will not occur under our assumption of $r_{I}>r_{0}$ and $w_{I}>w_{V}$.

Proposition 1. Under our assumption of $r_{I}>r_{0}$ and $w_{I}>$ $w_{V}$, all helpers the VoI will encounter have sufficient amount of data to transmit to the VoI.
Proof: Suppose when an arbitrary helper, denoted by $V_{i}, i=1,2 \ldots$, enters the coverage of $I_{2}$, it finds that there are already $n(n \geq 0)$ vehicles within $I_{2}$ 's coverage. Denote vehicles in front of $V_{i}$ (along the travel direction of $V_{i}$ ) by $V_{i+1}, V_{i+2} \ldots$ and distance between $V_{j}$ and $V_{j+1}$ is $l_{j}, j=1,2, \ldots$. If $n \leq N-1$, which implies $V_{i+N}$ is outside $I_{2}$ 's coverage, $V_{i}$ can immediately access a channel and start receiving data. Otherwise, $V_{i}$ has to wait until vehicle $V_{i+N}$ leaves the coverage of $I_{2}$ and free its channel resource. See Fig. 2 for an illustration.

According to the above two cases, the amount of data helper $V_{i}$ can receives from $I_{2}$, denoted by $D_{i}$, can be obtained as

$$
D_{i}=\min \left\{\frac{2 r_{I} w_{I}}{v_{2}}, \frac{S_{N_{i}} w_{I}}{v_{2}}\right\}, i=1,2, \ldots,
$$

here $S_{N_{i}}=\sum_{j=i}^{i+N-1} l_{j}$ is the distance between vehicle $V_{i}$ and vehicle $V_{i+N}$.

For the first helper VoI will encounter, without loss of generality, denote it as $V_{j}$, the amount of data it receives from $I_{2}$ is $D_{j}=\min \left\{\frac{2 r_{I} w_{I}}{v_{2}}, \frac{S_{N_{j}} w_{I}}{v_{2}}\right\}$ and the amount of data it transmits to the VoI is $D_{F_{j}}=\min \left\{\frac{2 r_{0} w_{V}}{v_{1}+v_{2}}, \frac{l_{j} w_{V}}{v_{1}+v_{2}}, D_{j}\right\}$, where $l_{j}$ is the distance between $V_{j}$ and origin when the VoI exit the coverage of $I_{1}$. As we have $S_{N_{j}}>l_{j}, r_{I}>r_{0}$ and $w_{I}>w_{V}$, it can be easily seen that $D_{j}>D_{F_{j}}$, which implies that the first helper the VoI will encounter has sufficient amount of data to transmit.

For an arbitrary helper the $\mathrm{VoI}$ will encounter excluding the first helper, denote it as $V_{i}$. If $V_{i}$ 's immediately preceding vehicle $V_{i+1}$ has sufficient data to transmit to the VoI during their $\mathrm{V} 2 \mathrm{~V}$ communication, the amount of data $V_{i}$ can transmit to the VoI during $\mathrm{V} 2 \mathrm{~V}$ communication, denoted by $D_{F_{i}}$, is given by:

$$
D_{F_{i}}=\min \left\{\frac{2 r_{0} w_{V}}{v_{1}+v_{2}}, \frac{l_{i} w_{V}}{v_{1}+v_{2}}, D_{i}\right\}, i=1,2, \ldots,
$$

As we have $S_{N_{i}} \geq l_{i}$ and further using the conditions that $r_{I}>r_{0}$ and $w_{I}>w_{V}$, it can be easily seen that $D_{i}>$ $D_{F_{i}}$ will always hold, which means for an arbitrary helper the VoI will encounter during one cycle, if its immediately preceding vehicle has sufficient data to transmit to the VoI, this arbitrary helper will also has sufficient data to transmit to the VoI. Therefore, the conclusion easily follows by recursion as we have shown above that the first helper will always have sufficient data to transmit.

To calculate $E\left[D_{V}\right]$, we need to obtain the expected $\mathrm{V} 2 \mathrm{~V}$ transmission time during $\mathrm{V} 2 \mathrm{~V}$ communication process in one cycle. As Proposition 1 above demonstrates, during the whole $\mathrm{V} 2 \mathrm{~V}$ communication period, the $\mathrm{VoI}$ can receive data as long as there exists helpers within its coverage. Thus, the problem of calculating the expected $\mathrm{V} 2 \mathrm{~V}$ transmission time in one cycle can be equally converted into a coverage process problem in which the total covered length are proportional to the total $\mathrm{V} 2 \mathrm{~V}$ communication time in our problem, where the proportionality constants are the relative speed between the 


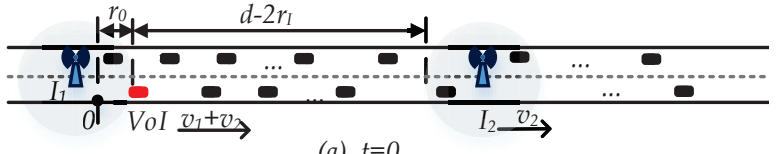

(a). $t=0$

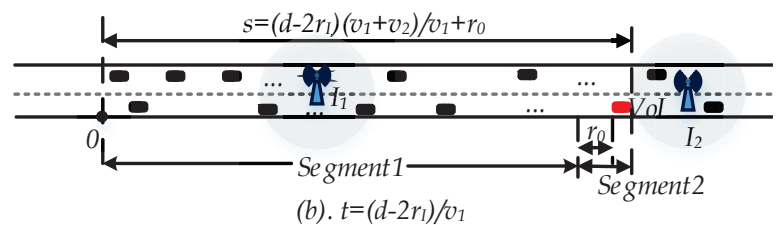

Fig. 3. An illustration of $\mathrm{V} 2 \mathrm{~V}$ communication between the $\mathrm{VoI}$ and helpers.

VoI and helpers. In the following, we will solve this coverage process problem.

2) $E\left[D_{V}\right]$ Calculation: To make the analysis easier, we change the coordinate system such that helpers become static (recall that we assume vehicles travel at constant speed), the $\mathrm{VoI}$ and infrastructure move at speeds of $v_{1}+v_{2}$ and $v_{2}$ respectively. In this way, when $t=0$, the displacement of $\mathrm{VoI}$ is $r_{0}$. The time instant the VoI enters $I_{2}$ 's coverage will be $t=\frac{d-2 r_{I}}{v_{1}}$ and its displacement at that time instant is

$$
s=\frac{\left(d-2 r_{I}\right)\left(v_{1}+v_{2}\right)}{v_{1}}+r_{0} .
$$

Thus, all vehicles in the opposite direction within this road segment $[0, s]$ are the helpers the VoI will encounter during its V2V communication process in one cycle. See Fig.3 for an illustration.

As an easy consequence of the total probability theorem, the expected data amount received from $\mathrm{V} 2 \mathrm{~V}$ communication in one cycle can be expressed as follows:

$$
E\left[D_{V}\right]=\sum_{y=0}^{\infty} E\left[D_{V} \mid Y=y\right] \cdot \operatorname{Pr}(Y=y),
$$

where $Y$ denotes the random number of helpers the VoI encounters during one cycle. Due to the Poisson distribution of vehicles (recall that helpers' vehicular density is $\rho_{2}$ ), we have

$$
P(Y=y)=\frac{\left(\rho_{2} s\right)^{y} e^{-\rho_{2} s}}{y !}, y=0,1,2, \ldots, \rho_{2}>0 .
$$

We denote the distance between the VoI and left boundary of $I_{2}$ 's coverage (the left boundary of $I_{2}$ 's coverage is also moving along with the movement of $\left.I_{2}\right)$ by $l(t)$ at time instant $t$, then the road segment $[0, s]$ can be divided into two segments, those outside $r_{0}$ of the left boundary of $I_{2}$ 's coverage (satisfies $l(t) \geq r_{0}$ ), denoted by Segment1 and those inside $r_{0}$ of the left boundary of $I_{2}$ 's coverage (satisfies $\left.l(t)<r_{0}\right)$, denoted by Segment 2 .

Conditioned on $Y=y$, the $y$ helpers are uniformly distributed in the above road segment $[0, s]$. Thus, when the VoI is within Segment1, the probability that at least one helper is in its coverage, denoted by $P_{1}$, can be obtained as

$$
P_{1}=1-\left(1-\frac{2 r_{0}}{s}\right)^{y} \text {. }
$$

When the VoI is at an arbitrary point in Segment2 (say when $\left.l(t)=z, z<r_{0}\right)$, the probability that at least one helper is in its coverage, denoted by $P_{2}$, is as follows:

$$
P_{2}=1-\left(1-\frac{r_{0}+z}{s}\right)^{y}, 0 \leq z<r_{0} .
$$

By summing up the above two cases, we have

$$
\begin{aligned}
E\left[D_{V} \mid Y=y\right] & =P_{1} \cdot \frac{\left(d-2 r_{I}-r_{0}\right) w_{V}}{v_{1}}+\int_{0}^{r_{0}} P_{2} \cdot \frac{w_{V} d z}{v_{1}} \\
& =\frac{c_{1} \cdot w_{V}}{v_{1}}+\frac{c_{2} \cdot w_{V}}{v_{1}(y+1)},
\end{aligned}
$$

here $c_{1}=\left(1-\left(1-\frac{2 r_{0}}{s}\right)^{y}\right)\left(d-2 r_{I}-r_{0}\right)+r_{0}$, and $c_{2}=$ $s \cdot\left[\left(\frac{s-2 r_{0}}{s}\right)^{y+1}-\left(\frac{s-r_{0}}{s}\right)^{y+1}\right]$.

By plugging equations (8) and (11) into (7) and simplifying it, we can obtain

$$
\begin{aligned}
E\left[D_{V}\right]= & \frac{\left(d-2 r_{I}-r_{0}\right) w_{V}}{v_{1}}\left(1-e^{-2 \rho_{2} r_{0}}\right) \\
& +\frac{w_{V} r_{0}}{v_{1}}+\frac{w_{V}\left(e^{-2 \rho_{2} r_{0}}-e^{-\rho_{2} r_{0}}\right)}{v_{1} \rho_{2}} .
\end{aligned}
$$

\section{Achievable Throughput}

From the above analysis, the achievable throughput can be calculated from equation (2), (3) and (12) as follows:

$$
\eta=\frac{2 r_{I} w_{I}+c \cdot w_{V}}{d}
$$

with $c=\left(d-2 r_{I}-r_{0}\right)\left(1-e^{-2 \rho_{2} r_{0}}\right)+r_{0}+\frac{e^{-2 \rho_{2} r_{0}}-e^{-\rho_{2} r_{0}}}{\rho_{2}}$.

\section{Simulation And Discussion}

In this section we use simulations conducted in Matlab to verify the accuracy of the analysis and establish the applicability of the theoretical analysis for more general scenarios beyond the ideal assumptions (e.g., unit disk model and constant channel condition) used in the analysis. Specifically, 50 infrastructure points are regularly deployed in the highway and their interval distance $d$ is varied from $2 \mathrm{~km}$ to $25 \mathrm{~km}$. The helpers' vehicular density $\rho_{2}$ varies from 0 to $0.1 \mathrm{veh} / \mathrm{m}$ and the speed of the VoI and helpers are $v_{1}=15 \mathrm{~m} / \mathrm{s}$ and $v_{2}=25 \mathrm{~m} / \mathrm{s}$ respectively. Infrastructure's and vehicles' radio ranges are varied from $400 \mathrm{~m}$ to $600 \mathrm{~m}$ and $200 \mathrm{~m}$ to $300 \mathrm{~m}$ (typical radio ranges using DSRC [6]) separately and constant transmission rates of $\mathrm{V} 2 \mathrm{I}$ and $\mathrm{V} 2 \mathrm{~V}$ communications are $w_{I}=5 \mathrm{Mb} / \mathrm{s}$ and $w_{V}=2.5 \mathrm{Mb} / \mathrm{s}$ respectively. Each infrastructure's channel number $N$ is 10. Each simulation is done 5000 times to get the average value.

Fig. 4 shows the comparison of the obtained analytical result with the simulation result and it is obvious that they are matched with each other, which demonstrates that the independence approximation adopted in section IV.A has negligible impact on the result and our final analytical result under this approximation is accurate.

Fig. 4 gives insight into the optimum choice of distance between infrastructure. It is obvious from Fig. 4 that when $d$ is increased, achievable throughput $\eta$ decreases. However, when $d$ increases beyond a certain threshold, e.g., $d=10 \mathrm{~km}$ in 


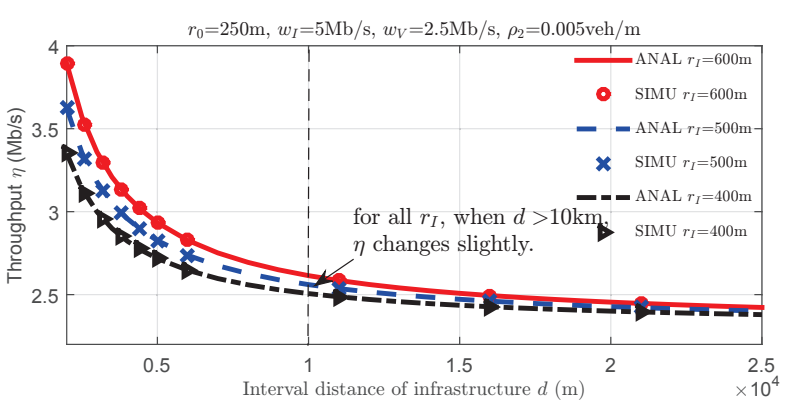

Fig. 4. A comparison of analytical and simulation results, with different $r_{I}$ equals to $600 \mathrm{~m}, 500 \mathrm{~m}$ and $400 \mathrm{~m}$ respectively. The helpers' vehicular density $\rho_{2}$ is $0.005 \mathrm{vhe} / \mathrm{m}$ and vehicles' radio range $r_{0}$ is $250 \mathrm{~m}$.

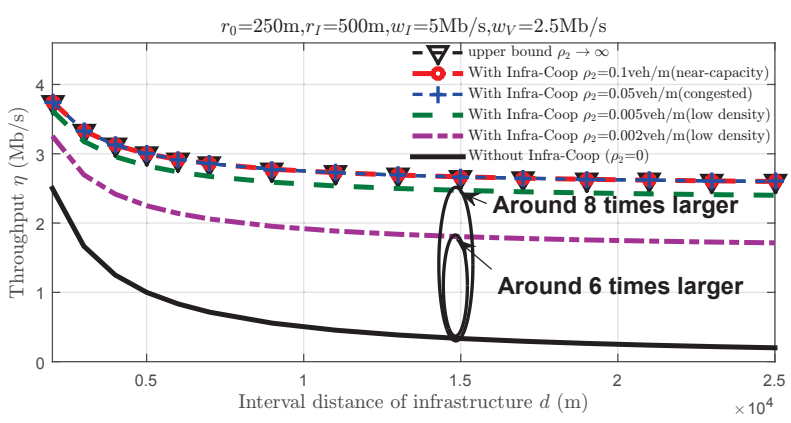

Fig. 5. A comparison between throughput achieved from systems with and without cooperative communication by setting $\rho$ as $\infty, 0.1 \mathrm{veh} / \mathrm{m}, 0.05 \mathrm{veh} / \mathrm{m}$, $0.005 \mathrm{veh} / \mathrm{m}, 0.002 \mathrm{veh} / \mathrm{m}$ and 0 respectively.

this case, an increase in $d$ has limited impact on the throughput achievable. This phenomenon can be explained by the fact that when $d$ is small, achievable throughput is mainly dominated by V2I communications because in this case, the amount of data received from $\mathrm{V} 2 \mathrm{~V}$ communications is relatively small compared with that received from V2I communications, especially when traffic density is low (here $\rho_{2}=0.005 \mathrm{veh} / \mathrm{m}$ ). However, with the rise of $d$, the increase of data received from V2V communications makes V2I communications' dominant impact subdued, which in turn leads to the subtle variation in $\eta$.

Fig. 5 demonstrates an important conclusion that our cooperative communication strategy can significantly improve the throughput even when helpers' vehicular density is low. It is shown that the throughput achieved from system utilizing cooperative communication with helpers' vehicular density $\rho_{2}=0.005 \mathrm{veh} / \mathrm{m}(0.002 \mathrm{veh} / \mathrm{m})$ is around $8(6)$ times larger than that without cooperative communication (equivalent to the case when there is no helpers, i.e., $\rho_{2}=0$ ), which shows the effectiveness of our proposed cooperative communication strategy.

Fig. 6 shows the relationship between $\eta$ and helpers' traffic density $\rho_{2}$. Importantly, we can see that higher traffic density is beneficial to the throughput because higher $\rho_{2}$ will enhance the connectivity of vehicular networks, which leads to higher chance of V2V communications. However, when $\rho_{2}$ increases beyond a certain threshold, e.g., $\rho_{2}=0.006 \mathrm{veh} / \mathrm{m}$ in this case,

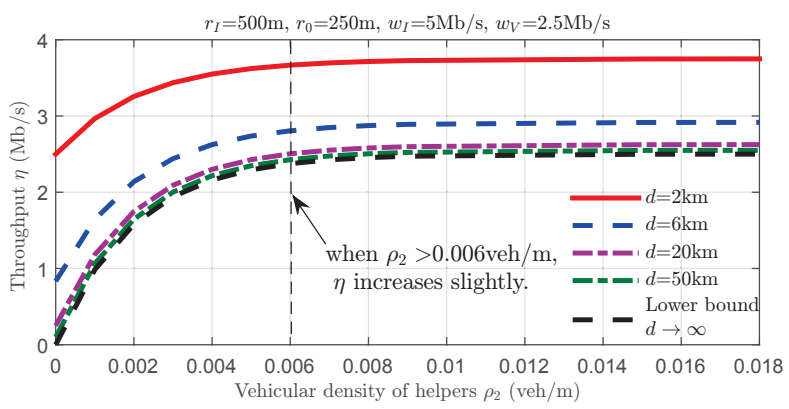

Fig. 6. Relationship between $\eta$ and helpers' vehicular density $\rho_{2}$ with different $d$ equals to $2 \mathrm{~km}, 6 \mathrm{~km}, 20 \mathrm{~km}, 50 \mathrm{~km}$ and tends to $\infty$ respectively.

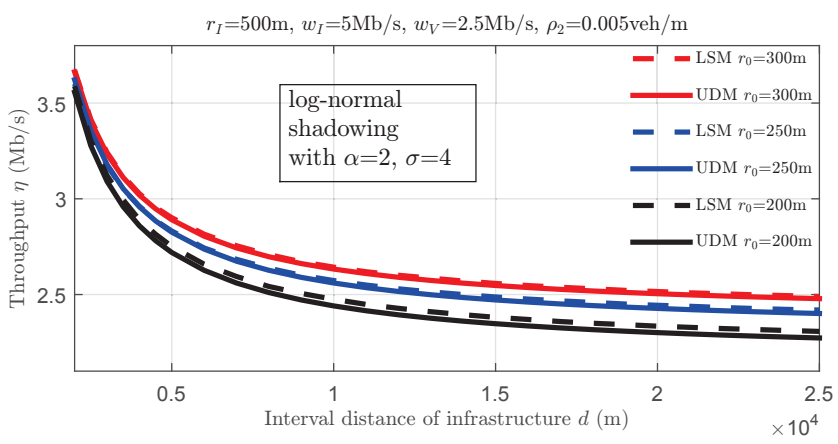

Fig. 7. A comparison between throughput achieved from unit disk model and log-normal shadowing model with path loss exponent $\alpha=2$ and standard variance $\sigma=4$.

a further increase in $\rho_{2}$ has only marginal impact on the achievable throughput. This is due to the fact that in our system model, helpers always have sufficient data to transmit to the VoI. Thus, when traffic density is large enough for the VoI to find at least one helper in its coverage at any time point, increasing traffic density (which will lead to more helpers within the VoI's coverage at one time) is no longer helpful to improve throughput because the VoI can only receive data from one vehicle at one time.

Fig. 7 gives the comparison of throughput achieved from unit disk model (labeled as UDM) and that from log-normal shadowing model (labeled as LSM), and shows that our analysis under unit disk model can be extended to log-normal shadowing model. The parameters of LSM are set as: path loss exponent $\alpha=2$ and standard deviation $\sigma=4$ [19], [20]. It is shown that the system under LSM has a slightly higher achievable throughput than that under UDM, which coincides with results in [20] that LSM is beneficial to information delivery in vehicular networks.

Fig. 8 compares throughput achieved from constant channel model with that from time-varying channel model, and shows that our analysis under constant channel model is applicable to a more realistic time-varying channel model which considers both fading and path loss. Specifically, for time-varying channel model, we adopt the model used in [21] that considers Rayleigh fading and path loss, from which the transmission rate is given by $w_{I}^{\prime}=B_{I} \log _{2}\left(1+P_{I}\left|\beta d_{i}^{-2}\right|^{2}\right)$ and $w_{V}^{\prime}=$ $B_{V} \log _{2}\left(1+P_{V}\left|\beta d_{i j}^{-2}\right|^{2}\right)$, with the bandwidth and transmit 


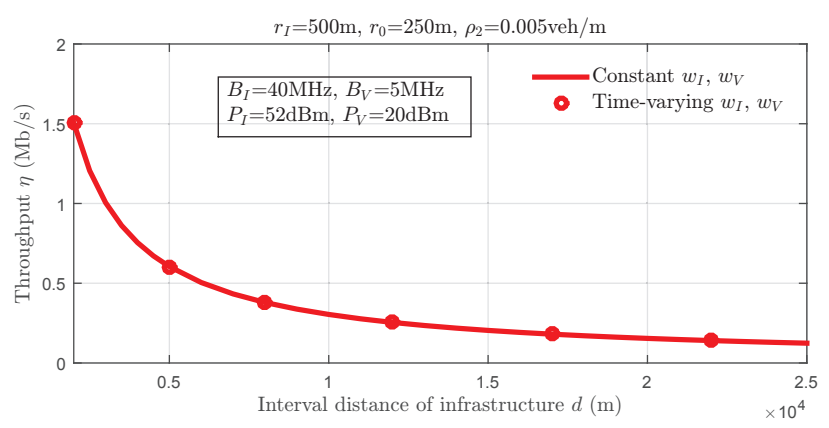

Fig. 8. A comparison between throughput achieved from constant channel model and time-varying channel model.

power of each infrastructure and vehicle are $B_{I}=40 \mathrm{MHz}$, $P_{I}=52 \mathrm{dBm}$ and $B_{V}=5 \mathrm{MHz}, P_{V}=20 \mathrm{dBm}$ [8] respectively. $\beta$ is the Gaussian random variable with mean 0 and variance 1 and $d_{i}, d_{i j}$ are the distances between vehicle and infrastructure, vehicle and vehicle when conducting V2I and V2V communications respectively. By dividing the total coverage length of the transmitter (infrastructure or vehicle) into $K$ (here we set $K=1000$ ) subtle segments, the average channel throughput $w_{I}$ and $w_{V}$ in time-varying channel model can be obtained by averaging the transmission rates of all segments. This obtained average throughput $w_{I}$ and $w_{V}$ are then used in our constant channel model. It is obvious from Fig. 8 that the achievable throughput from the above two channel models match with each other. This phenomenon can be explained by equation (13) which shows that the achievable throughput is a linear function of $w_{I}$ and $w_{V}$. Then according to Jensen's Inequality, we have $E\left[\eta\left(w_{I}, w_{V}\right)\right]=\eta\left(E\left[w_{I}\right], E\left[w_{V}\right]\right)$, which shows that for time-varying channels, the time-varying values of $w_{I}^{\prime}$ and $w_{V}^{\prime}$ can be replaced by the respective time-averaged throughput of V2I and V2V communications and our analysis still applies.

\section{CONCLUSION AND FutURE WORK}

This paper proposed a cooperative communication strategy for vehicular networks by utilizing V2I communications, V2V communications and the mobility of vehicles to facilitate the transmission. From our analytical and numerical results, we concluded that this cooperative strategy can effectively improve the achievable throughput of vehicular networks even when traffic density is rather low. Our analysis can also be extended to more realistic models such as log-normal shadowing model and time-varying channel model considering fading and path loss. Moreover, our result can be used as a guide of optimum design and deployment of infrastructure in vehicular networks. In the future we will consider a general case that vehicles (helpers) do not have sufficient amount of data to transmit. Further, multiple vehicles of interest will also be considered.

\section{ACKNOWLEDGMENT}

This research is supported by Australian Research Council (ARC) Discovery projects DP110100538 and DP120102030 and Chinese National Science Foundation project 61428102.

\section{REFERENCES}

[1] Y. Li, X. Zhu, D. Jin and D. Wu, "Multiple Content Dissemination in Roadside-Unit-Aided Vehicular Opportunistic Networks," IEEE Trans. Veh. Technol., vol. 63, no. 8, pp. 3947-3956, Oct. 2014.

[2] S. Al-Sultan, M. M. Al-Doori, A. H. Al-Bayatti, and H. Zedan, "A Comprehensive Survey on Vehicular Ad Hoc Network," Journal of Network and Computer Applications, vol. 37, pp. 380-392, 2014.

[3] N. Lu, N. Cheng, N. Zhang, X. Shen and J. W. Mark, "Connected Vehicles: Solutions and Challenges," IEEE Internet Things J., vol 1, no. 4, pp. 289-299, Aug. 2014.

[4] M. Khabazian, S. Aissa, and M. Mehmet-Ali, "Performance Modeling of Safety Messages Broadcast in Vehicular Ad Hoc Networks", IEEE Trans. Intell. Transp. Syst., vol. 14, no. 1, pp. 380-387, Mar, 2013.

[5] A. B. Reis, S. Sargento, F. Neves, and O. K. Tonguz, "Deploying Roadside Units in Sparse Vehicular Networks: What Really Works and What Does Not," IEEE Trans. Veh. Technol., vol. 63, no. 6, pp. 27942806, Jul. 2014.

[6] Z. Haibo, L. Bo, T. H. Luan, H. Fen, G. Lin, L. Ying, Q. Yu, and X. Shen, "ChainCluster: Engineering a Cooperative Content Distribution Framework for Highway Vehicular Communications," IEEE Trans. Intell. Transp. Syst., vol. 15, no.6, pp. 2644-2657, Dec. 2014.

[7] W. Zhu, D. Li, and W. Saad, "Multiple Vehicles Collaborative Data Download Protocol via Network Coding," IEEE Trans. Veh. Technol., vol. 64, no. 4, pp. 1607-1619, Apr. 2015.

[8] K. Zheng, F. Liu, Q. Zheng, W. Xiang and W. Wang, "A Graph-Based Cooperative Scheduling Scheme for Vehicular Networks," IEEE Trans. Veh. Technol., vol. 62, no. 4, pp. 1450-1458, May. 2013.

[9] M. Garetto, and E. Leonardi, "Restricted Mobility Improves DelayThroughput Tradeoffs in Mobile Ad Hoc Networks," IEEE Trans. Inf. Theory, vol 56, no. 10, pp. 5016-5029, Oct. 2010.

[10] R. Kim, H. Lim, and B. Krishnamachari, "Prefetching-Based Data Dissemination in Vehicular Cloud Systems," IEEE Trans. Veh. Technol. vol. PP, no. 99, pp. 1-1, Jan. 2015.

[11] A. Agarwal, D. Starobinski, and T. D. C. Little, "Phase Transition of Message Propagation Speed in Delay-Tolerant Vehicular Networks" IEEE Trans. Intell. Transp. Syst., vol. 13, pp. 249-263, Mar. 2012.

[12] N. Wisitpongphan, B. Fan, P. Mudalige, V. Sadekar, and O. Tonguz, "Routing in Sparse Vehicular Ad Hoc Wireless Networks," IEEE J. Sel. Areas Commun., vol. 25, no. 8, pp. 1538-1556, Oct. 2007.

[13] W. Zhang, Y. Chen, Y. Yang, X. Wang, Y. Zhang, X. Hong and G. Mao, "Multi-Hop Connectivity Probability in Infrastructure-Based Vehicular Networks," IEEE J. Sel. Areas Commun., vol. 30, no. 4, pp. 740-747, May. 2012.

[14] Z. Zhang, G. Mao, and B. D. O. Anderson, "Stochastic Characterization of Information Propagation Process in Vehicular Ad hoc Networks," IEEE Trans. Intell. Transp. Syst., vol. 15, no. 1, pp. 122-135, Feb. 2014.

[15] W. Wei, S. S. Liao, L. Xin, and J. S. Ren, "The Process of Information Propagation Along a Traffic Stream Through Intervehicle Communication,' IEEE Trans. Intell. Transp. Syst, vol. 15, no. 1, pp. 345-354, Feb. 2014.

[16] W. Tan, W. Lau, O. Yue and T. Hui, "Analytical Models and Performance Evaluation of Drive-thru Internet Systems," IEEE J. Sel. Areas Commun., vol. 29, no. 1, pp. 207-222, Jan. 2011.

[17] P. Wang, G. Mao, Z. Li, X. Ge and B. O. Anderson, "Network Coding based Wireless Broadcast with Performance Guarantee," IEEE Trans. Wireless Commun., Vol. 14, No. 1, pp. 532 - 544, Jan. 2015.

[18] R. G. Gallager, Stochastic Processes: Theory for Applications. Cambridge University Press, 2013.

[19] T. S. Rappaport, Wireless Communications: Principles and Practice, 2nd Edition. Prentice Hall, 2001.

[20] Z. Zhang, G. Mao, T. Han and B. D. O. Anderson, "Cooperative Information Forwarding in Vehicular Networks Subject to Channel Randomness," in Proc. IEEE ICC, 2014.

[21] T. Wang, L. Song and Z. Han, "Coalitional Graph Games for Popular Content Distribution in Cognitive Radio VANETs," IEEE Trans. Veh. Technol., vol. 62, no. 8, pp. 4010-4019. Oct. 2013. 